\title{
ANTI-HYPERGLYCEMIC AND ANTI-HYPERLIPIDEMIC POTENTIAL OF A POLYHERBAL PREPARATION "DIABEGON" IN METABOLIC SYNDROME SUBJECT WITH TYPE 2 DIABETES
}

\section{Dhananjay Yadav ${ }^{1,2 *}$, Arvind Tiwari ${ }^{2}$, Meerambika Mishra ${ }^{3}$, Senthil S Subramanian ${ }^{2}$, Usha Singh Baghel ${ }^{4}$, Sunil Mahajan $^{2}$, P.S.Bisen ${ }^{2}$, GBKS Prasad $^{2}$}

\author{
${ }^{1}$ Department of Internal Medicine, Yonsei University Wonju College of Medicine, Wonju, South Korea, ${ }^{2}$ SOS in \\ Biochemistry Jiwaji University, Gwalior (M.P), India. ${ }^{3}$ School of Life Sciences, Sambalpur University, Jyoti Vihar, \\ Burla(Odisha), ${ }^{4}$ School of Environmental Science, JNU-New Delhi \\ *Email: dhanyadav16481@gmail.com
}

\begin{abstract}
Background: In the present study, "Diabegon" a poly-herbal preparation, with hypoglycemic activity, was evaluated for its preventive effect in metabolic syndrome subjects with type 2 diabetes and also to reveal its side effects, on liver and kidney.

Materials and Methods: Type 2 diabetic subjects with metabolic syndrome ( $\mathrm{N}=58)$ were categorized on the basis of age and fasting blood glucose. The grouping was as follows: Group I (35-50 yrs), Group II (51-65 yrs), Group III >65 yrs, Group IV FBS <145.9, Group V FBS >145. Each group was administered $4 \mathrm{gm}$ of diabegon daily. Blood glucose levels, lipid profile, liver and kidney function of the subjects were regularly monitored within 3 months of interval to 18 months.

Results: The reduction in fasting blood glucose level ranged from $12.3 \%(\mathrm{P}<0.05)$ to $42 \%(\mathrm{P}<0.001)$ after 18 month of therapy whereas in postprandial blood glucose, the decrease ranged from $28 \%(\mathrm{P}<0.05)$ to $32 \%(\mathrm{P}<0.05)$ after 18 month of therapy. Overall reductions in the individual parameters of the metabolic syndrome subjects were significantly higher in Group I. Cholesterol level decreased from $11 \%$ to $27.2 \%$ ( $\mathrm{P}<0.001$ ), triglyceride levels decreased from $24 \%$ to $55 \%$, VLDL and LDL levels reduced by $60 \%$ \& 54\% respectively after 18 months of therapy. The HDL-C level increased in all groups. Moreover, diabegon administration for 1.5 years exhibited no alteration in liver and kidney function tests, which indicate its non-toxicity.

Conclusion: Our study suggests that diabegon could be included as a preventive treatment in metabolic syndrome subjects with type 2 diabetes especially for long term treatment as it efficiently shows anti-hyperglycemic and anti-lipidemic effects with no adverse impacts on the liver and kidney.
\end{abstract}

Key words: Metabolic syndrome, Type 2 diabetes, Diabegon, Polyherbal preparation.

\section{Introduction}

Metabolic syndrome combines a group of several risk factors like insulin resistance, hypertension, dyslipidemia and obesity. T2 diabetes and CVD play an important role in the increasing the burden of death because of the severity and complication of the disease. Studies reveal that metabolic syndrome with type 2 diabetes leads to more cardiovascular events than only metabolic syndrome (Sone et al., 2009; Wilson et al., 2005). So these subjects require more attention on health and also needed a follow-up or intervention study.

There are many studies that focus on the prevalence of metabolic syndrome in different populations. Some of those observed in respect of prevalence that ranges from 13-30\% and 70-80\% among the Caucasian non-diabetic (Ford et al., 2002; Novakovic et al., 2001) and diabetic (Balkau et al., 2002) populations, respectively. According to Reaven et al genetically determined insulin resistance in a setting of suitable environmental factors is the pivotal pathogenic mechanism underlying the metabolic syndrome.

The clinical management of T 2 diabetic patients with the metabolic syndrome is to reduce the risk of further cardiovascular disease. It is associated with the drug therapy for the metabolic syndrome imply on amendment of the individual risk factors i.e. hypertension, dyslipidemia, adiposity and hyperglycemia (Eckel et al., 2005; Grundy, 2006). Hyperglycemia associated with lipid disorder leads to dysfunction of liver, skeletal muscle and adipose tissue later it passes to vital organ of the body like kidney, eyes and nervous system. Hence metabolic syndrome could be a prognostic marker for the severity of complication in T2 diabetes. Therefore our study focuses on metabolic syndrome subjects with T2 diabetes. A lot of pharmaceutical drug has been available for the treatment of metabolic syndrome and T2 diabetes but none of them are well suited in every respect of side effects. Sulfonylurea and biguanides used for treatment of diabetes are highly expensive or have undesirable side effects or contraindication (Halim and Hussain, 2002; Chen et al., 2003). Drugs like sulfonylurea shows some toxic effect like heartburn, vomiting, skin rashes etc (Seshiah, 2004). Biguanides (metformin) on long-term consumption may generate gastrointestinal effects, anorexia, vomiting, and $B_{12}$ malabsorbtion.

Hence there arises an urgent need to identify natural resources and study their potential on different identified targets in order to develop them as new anti-diabetic therapeutics (oral hypoglycemic agents and insulin) for the treatment of diabetes mellitus. Many experimental and clinical trials have been carried out for anti-diabetic property of herbal preparation in diabetes (Babu et al., 2004; Bhandari et al., 2005; Tongia et al., 2004). Even WHO suggested the evaluation of the potential of plants as effective therapeutic agents, especially in areas where we lack safe modern drugs WHO (World Health Organization Geneva 1994).

Based on the ancient Indian Ayurvedic text that describes the cure for diabetes, a polyherbal mixure is prepared by Dindayal Aushdhi Ltd. Gwalior namely Diabegon ${ }^{\circledR}$. The diabegon, a polyherbal formulation of containing 18 plant extracts with known hypoglycemic activity is shown in our previous study in animal by Hariom et al., 2007. Hariom et al. evaluated the hypoglycemic effect of diabegon in animal model but the long term follow-up treatment has not been evaluated with special reference to metabolic syndrome. Hence the present study assessed therapeutic potential of polyherbal preparation "Diabegon" in metabolic syndrome subjects with type 2 diabetes and also study revealed its side effect, on liver, kidney.

\section{Material and methods Selection of subjects}

Type 2 diabetes with metabolic syndrome subjects was randomly selected from different age groups from our weekend diabetic clinic run in the school of studies in Biotechnology at Jiwaji University Gwalior. The time period was in between (2007-2009). All the recruited subjects expressed 
their willingness to participate in the study. The study group consisted of $58 \mathrm{~T} 2$ diabetic subjects with metabolic sydrome on the basis of age and fasting blood sugar (FBS). First three groups divided accordingly to age and other two groups were based on lower (FBS $<145.9 \mathrm{mg} / \mathrm{dl})$ and higher blood group (FBS $>145.9 \mathrm{mg} / \mathrm{dl}$ ).

Group I NIDDM age group (35-50) N=10, Group II NIDDM age group (51-65) N=34, Group III NIDDM age group >65 N=14, Group IV NIDDM FBS $<145.9$ (N=43), Group V NIDDM FBS $>145.9(\mathrm{~N}=15)$.

A baseline questionnaire requested information on diet pattern, lifestyle, known risk factors for diabetes and socioeconomic background etc. The study design and experimental protocols were approved by the Institutional Human Ethics Committee. The drug was prescribed by Ayurvedic physician personally to every subject at a dose of $4 \mathrm{Gms}$. (twice a day) with water as prescribe in Ayurvedic Pharmacopoeia of India. The treatment was monitored at about one and half year and followed by monthly checkup. The subjects were kept away from any other type of anti-diabetic medication.

\section{Anthropometrical and biochemical parameters}

Selected anthropometric variables like Height, weight and waist circumferences were measured with the subject barefooted and lightly dressed. Table 1 showed the different groups characterized on the age group and fasting blood glucose level

\section{Sample collection and biochemical estimations}

Fasting blood glucose and lipid profile were measured at baseline and followed up every month till 18 month of therapy. The fasting blood glucose level was represented at 3 month intervals and lipid profile was represented in 6 months interval. The blood glucose level was determined by glucose oxidase-peroxidase method using a kit Monozyme India limited, Ahmadabad (Trinder, 1969). Total cholesterol, triglyceride and HDLcholesterol were estimated by CHOD-PAP (Stockbridge et al., 1989), triglycerides (Fossati and Prencipe, 1982) and HDL- Cholesterol (Lopes-virella et al., 1977) was estimated by spectrophotometric assays employing commercially available kits. LDL and VLDL were calculated from Freidewald's formula. Kidney function was monitored by studying the changes in urea using the (Fawcett, 1960) uric acid (Fossati, 1980)) and creatinine (Bowers, 1980). Liver function was assessed by monitoring bilirubin (modified Jendrassik and Grof's method, 1938), serum glutamate oxaloacetate transaminase (SGOT) and serum glutamate pyruvate transaminase (SGPT) (modified International Federation of Clinical Chemistry [IFCC] method, Burtis and Ashwood, 1999).

\section{Diagnostic criteria of the metabolic syndrome}

NCEP-ATP III criteria were used for the diagnosis of metabolic syndrome (NCEP JAMA, 2001).

\section{Statistical Analysis}

The results were expressed as mean \pm standard deviation. The data obtained from the experiments were analyzed using one-way ANOVA (Bonferroni t-test) employing Sigma Stat, statistical software, version 1.0 (Jandal corporation, USA). For computation of data, software application programmes like Microsoft Excel, Sigma Direct. The values were tested for significance at $\mathrm{P}<0.001, \mathrm{P}<0.05$.

\section{Results}

Anthropometric parameters of Non Insulin Dependent Diabetes Mellitus subjects with metabolic sydrome having different age group selected for diabegon Study

The Subject of either sex was selected for studies which were grouped into five groups as shown in Table 1. The groups were studied with respect to anthropometric parameters; age, duration of disease, waist circumference, BMI, systolic blood pressure, diastolic blood and pulse rate. On comparing the age of Group II with Group I significance was observed. The age Group was found to be significant (P $\leq 0.001)$ when comparisons were made between Group I\&III Group II\& IIIGroup, V\&III Group and IV\&III Groups. In age group significance (P $\leq 0.001)$ was observed when comparisons were made between II \&I IV\&I Groups. Systolic blood pressure was significant (P $\leq .05)$ when group I compared with III, V.

Table 1:.Anthropometric parameters of Non Insulin Dependent Diabetes Mellitus subjects with MetS having different age group selected for Diabegon Study

\begin{tabular}{|c|c|c|c|c|c|c|c|}
\hline Groups & Age (Year) & Duration of dis. (Year) & $\begin{array}{l}\text { Waist Circ } \\
\text { (Inches) }\end{array}$ & BMI $(\mathrm{Kg} / \mathrm{m} 2)$ & \begin{tabular}{|l} 
Systolic \\
$(\mathrm{mmHg})$
\end{tabular} & $\begin{array}{l}\text { Diastolic } \\
(\mathrm{mmHg})\end{array}$ & Pulse \\
\hline Group I $(35-50) \mathrm{N}=10$ (a) & $46 \pm 4.3$ & $5.5 \pm 4.6$ & $37.17 \pm 3$ & $27.0 \pm 4.1$ & $127 \pm 9.9$ & $76.9 \pm 6.2$ & $89.37 \pm 8.9$ \\
\hline Group II (51-65) N=34 (b) & $58.3 \pm 3.6 \mathrm{a}^{* *}$ & $4.3 \pm 2$ & $36.6 \pm 3.9$ & $25.9 \pm 3.6$ & $134.29 \pm 17$ & $74.2 \pm 7.2$ & $85.71 \pm 8.6$ \\
\hline Group III $>65 \mathrm{~N}=14$ (c) & $70 \pm 5\left(\mathrm{a}^{* *}, \mathrm{~b}^{* *} \mathrm{~d} * *, \mathrm{e}^{* *}\right)$ & $9.1 \pm 6.3$ & $38.0 \pm 7.3$ & $23.8 \pm 4.5$ & $140.7 \pm 18.9 \mathrm{a}^{*}$ & $72.8 \pm 8.6$ & $82.99 \pm 8.9$ \\
\hline $\begin{array}{l}\text { CG- IV FBS }<145.9 \text { (d) } \\
N=43\end{array}$ & $59.4 \pm 7.4 \mathrm{a}^{* *}$ & $5.3 \pm 4$ & $37.9 \pm 5.2$ & $26.0 \pm 3.9$ & $136.0 \pm 17.1$ & $74.4 \pm 7$ & $84.5 \pm 9.1$ \\
\hline $\begin{array}{l}\text { CG-V FBS>145.9 (e) } \\
N=15\end{array}$ & $57.7 \pm 12.1 \mathrm{a}^{*}$ & $6.53 \pm 5.2$ & $35.3 \pm 3.3$ & $24.2 \pm 4.3$ & $138.9 \pm 13.3 \mathrm{a}^{*}$ & $77.1 \pm 8.3$ & $88.9 \pm 7.7$ \\
\hline
\end{tabular}


http://dx.doi.org/10.4314/ajtcam.v11i2.4

Tables 2: Effect of Diabegon® on blood glucose level in MetS with Non-insulin dependent diabetes mellitus subjects of different age groups (N=58)

\begin{tabular}{|c|c|c|c|c|c|c|c|c|c|c|c|c|c|c|}
\hline \multirow[t]{2}{*}{ (Groups) } & \multicolumn{7}{|c|}{ Fasting blood glucose $(\mathrm{mg} / \mathrm{dl})$} & \multicolumn{7}{|c|}{ Post-prandial blood glucose $(\mathrm{mg} / \mathrm{dl})$} \\
\hline & Initial (a) & $\begin{array}{l}3 \text { month of } \\
\text { therapy (b) }\end{array}$ & $\begin{array}{l}6 \text { month } \\
\text { of therapy } \\
\text { (c) }\end{array}$ & $\begin{array}{l}9 \text { month } \\
\text { of therapy } \\
\text { (d) }\end{array}$ & $\begin{array}{l}12 \text { month } \\
\text { of therapy } \\
\text { (e) }\end{array}$ & $\begin{array}{l}15 \text { month } \\
\text { of therapy } \\
\text { (f) }\end{array}$ & $\begin{array}{l}18 \text { month } \\
\text { of therapy } \\
\text { (g) }\end{array}$ & Initial(a) & $\begin{array}{l}3 \text { month of } \\
\text { therapy (b) }\end{array}$ & $\begin{array}{l}6 \text { month } \\
\text { of therapy } \\
\text { (c) }\end{array}$ & {$\left[\begin{array}{l}9 \text { month } \\
\text { of therapy } \\
\text { (d) }\end{array}\right.$} & $\begin{array}{l}12 \text { month } \\
\text { of therapy } \\
\text { (e) }\end{array}$ & $\begin{array}{l}15 \text { month } \\
\text { of therapy } \\
\text { (f) }\end{array}$ & $\begin{array}{l}18 \text { month } \\
\text { of therapy } \\
(\mathrm{g})\end{array}$ \\
\hline \begin{tabular}{|l} 
Group I \\
$(35-50)$, \\
$\mathrm{N}=10$
\end{tabular} & $160.3 \pm 33.9$ & $150 \pm 50.1$ & $142.7 \pm 55.8$ & $\begin{array}{l}120.5 \pm 38.2 \\
a^{*}\end{array}$ & $\begin{array}{l}117.8 \pm 38.1 \\
a^{*}\end{array}$ & $\begin{array}{l}118.6 \pm 41.6 \\
a^{*}\end{array}$ & $\begin{array}{l}122.1 \pm 27.3 \\
a^{*}\end{array}$ & $238.6 \pm 50.4$ & $218.8 \pm 82.3$ & $210.6 \pm 80.5$ & $\begin{array}{l}193.2 \pm 75.2 \\
a^{*}\end{array}$ & {$\left[\begin{array}{l}161.2 \pm 67.2 \\
a^{*}\end{array}\right.$} & $\begin{array}{l}173.7 \pm 50.2 \\
a^{*}\end{array}$ & {$\left[\begin{array}{l}185.3 \pm 30.3 \\
a^{*}\end{array}\right.$} \\
\hline \begin{tabular}{|l} 
Group II \\
$(51-65)$, \\
$\mathrm{N}=34$
\end{tabular} & $139.7 \pm 35.2$ & $134.1 \pm 48$ & $\begin{array}{l}116.4 \pm 5.5 \\
a^{* *} b^{*}\end{array}$ & $\begin{array}{l}111.8 \pm 19 \\
a^{* *} b^{*}\end{array}$ & $\begin{array}{l}110 \pm 23.2 \\
a^{* *} b^{*}\end{array}$ & $\begin{array}{l}114.2 \pm 25.4 \\
a^{* *} b^{*}\end{array}$ & $\begin{array}{l}102.5 \pm 34.3 \\
a * * b * c * d *\end{array}$ & $209.1 \pm 53.1$ & $183.2 \pm 70.7$ & {$\left[\begin{array}{l}185.6 \pm 20.1 \\
a^{*}\end{array}\right.$} & $\begin{array}{l}157 \pm 44.8 \\
a * c^{*}\end{array}$ & $\begin{array}{l}168.6 \pm 84.2 \\
\text { a* }\end{array}$ & $\begin{array}{l}164.2 \pm 34.6 \\
a * c^{*} *\end{array}$ & $\begin{array}{l}171.9 \pm 69.5 \\
a^{*}\end{array}$ \\
\hline $\begin{array}{l}\text { Group III } \\
>65, \mathrm{~N}=14\end{array}$ & $130.5 \pm 34$ & $127.8 \pm 43.2$ & $131.8 \pm 12.8$ & $123 \pm 50.8$ & $133.4 \pm 29.7$ & $124.9 \pm 30.7$ & $\begin{array}{l}114.4 \pm 26.7 \\
c^{*}\end{array}$ & $219.3 \pm 74$ & $185.8 \pm 79$ & $197.4 \pm 20.8$ & $193.6 \pm 77.5$ & $\begin{array}{l}171.5 \pm 25.4 \\
a * c^{*}\end{array}$ & $181.4 \pm 34.8$ & $\begin{array}{l}162.1 \pm 40.3 \\
a * c^{*}\end{array}$ \\
\hline \begin{tabular}{|l|} 
CB-IV, \\
N=43 \\
FBS $<145.9$ \\
\end{tabular} & $116 \pm 19.5$ & $119.6 \pm 37.8$ & $120.1 \pm 18.8$ & $110.5 \pm 43.8$ & $110.4 \pm 39.3$ & $110.7 \pm 21.5$ & $\begin{array}{l}106.6 \pm 27 \\
c^{*}\end{array}$ & $189.8 \pm 28.6$ & $\begin{array}{l}161.5 \pm 39.3 \\
a * *\end{array}$ & $\left\{\begin{array}{l}177.2 \pm 30.8 \\
b^{*}\end{array}\right.$ & $\begin{array}{l}163.9 \pm 67 \\
a^{*}\end{array}$ & $174.6 \pm 88$ & $170.3 \pm 61.2$ & {$\left[\begin{array}{l}164.4 \pm 52.3 \\
a^{*}\end{array}\right.$} \\
\hline \begin{tabular}{|l} 
CB-V, \\
$N=15$ \\
FBS $>145.9$
\end{tabular} & $189.2 \pm 19.2$ & $174 \pm 77.3$ & $\begin{array}{l}139.8 \pm 25.4 \\
a * * b *\end{array}$ & $\begin{array}{l}118.2 \pm 24.7 \\
a^{* *} b^{*} c^{*}\end{array}$ & $\begin{array}{l}130.6 \pm 34.1 \\
a^{* *} c^{*}\end{array}$ & $\begin{array}{l}162.9 \pm 45.6 \\
a * d * e^{*}\end{array}$ & $\begin{array}{l}110.2 \pm 36.1 \\
a^{* *} b * * c * f *\end{array}$ & $249.6 \pm 46.1$ & $224 \pm 80.5$ & $234.3 \pm 53$ & $218.6 \pm 76.5$ & $\begin{array}{l}211.1 \pm 50.9 \\
a^{*}\end{array}$ & $\begin{array}{l}192.5 \pm 8.84 \\
a^{* *} c^{*}\end{array}$ & $\begin{array}{l}196 \pm 69.8 \\
a^{*}\end{array}$ \\
\hline
\end{tabular}

Table 3: Effect of Diabegon ${ }^{\circledR}$ on blood cholesterol, triglyceride \& HDL-C in MetS with Non-insulin dependent diabetes mellitus subjects of different age groups (N=58)

\begin{tabular}{|c|c|c|c|c|c|c|c|c|c|c|c|c|}
\hline \multirow[b]{2}{*}{ (Group) } & \multicolumn{4}{|c|}{ Total Cholesterol (mg/dl) } & \multicolumn{4}{|c|}{ Triglyceride $(\mathrm{mg} / \mathrm{dl})$} & \multicolumn{4}{|c|}{ HDL-C (mg/dl) } \\
\hline & Initial (a) & $\begin{array}{l}6 \text { month of } \\
\text { therapy (b) }\end{array}$ & $\begin{array}{l}12 \text { month } \\
\text { of } \\
\text { therapy(c) }\end{array}$ & $\begin{array}{l}18 \text { month } \\
\text { of therapy } \\
\text { (d) }\end{array}$ & Initial (a) & $\begin{array}{l}6 \text { month of } \\
\text { therapy }(b)\end{array}$ & $\begin{array}{l}12 \text { month } \\
\text { of } \\
\text { therapy(c) }\end{array}$ & $\begin{array}{l}18 \text { month } \\
\text { of therapy } \\
\text { (d) }\end{array}$ & Initial (a) & $\begin{array}{l}6 \text { month } \\
\text { of } \\
\text { therapy } \\
\text { (b) }\end{array}$ & $\begin{array}{l}12 \text { month } \\
\text { of } \\
\text { therapy(c) }\end{array}$ & $\begin{array}{l}18 \text { month } \\
\text { of therapy } \\
\text { (d) }\end{array}$ \\
\hline $\begin{array}{l}\text { Group I } \\
(35-50) \\
\mathrm{N}=10\end{array}$ & $197.9 \pm 19.2$ & $\begin{array}{l}161.7 \pm 47.8 \\
a^{*}\end{array}$ & $\begin{array}{l}139.9 \pm 31.4 \\
a^{* *}\end{array}$ & $\begin{array}{l}147.1 \pm 17.6 \\
a^{* *}\end{array}$ & $281.4 \pm 93.9$ & $\begin{array}{l}192.5 \pm 31 \\
a^{*}\end{array}$ & $\begin{array}{l}110.06 \pm 8.8 \\
a^{* *} b * *\end{array}$ & $\begin{array}{l}126.3 \pm 37.8 \\
a * * b^{* *}\end{array}$ & $27.9 \pm 1.43$ & $\begin{array}{l}36.7 \pm 5.3 \\
a * *\end{array}$ & $39.5 \pm 16.7$ & $\begin{array}{l}54.5 \pm 23 \\
a * b *\end{array}$ \\
\hline $\begin{array}{l}\text { Group II } \\
(51-65) \\
, \mathrm{N}=34\end{array}$ & $167.4 \pm 20.1$ & $\begin{array}{l}183.5 \pm 42.1 \\
a^{*}\end{array}$ & $\begin{array}{l}153.5 \pm 23.2 \\
a * b * *\end{array}$ & $\begin{array}{l}148.9 \pm 44.8 \\
a^{*} b^{*}\end{array}$ & $169.3 \pm 29.4$ & $\begin{array}{l}120.3 \pm 56.7 \\
a^{* *}\end{array}$ & $\begin{array}{l}121 \pm 16 \\
a^{* *}\end{array}$ & $\begin{array}{l}111.7 \pm 47.6 \\
a^{* *}\end{array}$ & $44 \pm 10.4$ & $\begin{array}{l}32.2 \pm 5.8 \\
a^{* *}\end{array}$ & $\begin{array}{l}42.2 \pm 21.5 \\
b^{*}\end{array}$ & $\begin{array}{l}51.9 \pm 15 \\
a * b * *\end{array}$ \\
\hline $\begin{array}{l}\text { Group III } \\
>65, N=14\end{array}$ & $176.7 \pm 10.6$ & $163.3 \pm 65.8$ & $163.1 \pm 17.8$ & $151 \pm 43 \backslash \mathrm{a}^{*}$ & $133.5 \pm 33.4$ & $\begin{array}{l}157.4 \pm 14.4 \\
a *\end{array}$ & $136.8 \pm 43.4$ & $\begin{array}{l}101.2 \pm 30 \\
a * b * * c *\end{array}$ & $40.7 \pm 11.6$ & $41.7 \pm 8.1$ & $40.95 \pm 5.9$ & $51.2 \pm 25$ \\
\hline \begin{tabular}{|l|} 
CB-IV, \\
$\mathrm{N}=43$ \\
FBS $<145.9$
\end{tabular} & $158.9 \pm 18.3$ & $166.7 \pm 50.4$ & $156 \pm 57.7$ & $\begin{array}{l}144.7 \pm 39.4 \\
a^{*} b^{*}\end{array}$ & $158 \pm 80.6$ & $131.8 \pm 49.6$ & $\begin{array}{l}114.6 \pm 50.9 \\
a^{*}\end{array}$ & $\begin{array}{l}114 \pm 59 \\
a^{*}\end{array}$ & $41.4 \pm 13.3$ & $\begin{array}{l}35.2 \pm 6.2 \\
a^{*}\end{array}$ & $40.5 \pm 16.9$ & $\begin{array}{l}52.7 \pm 19.3 \\
a * b^{* *} c^{* * *}\end{array}$ \\
\hline \begin{tabular}{|l|}
$\mathrm{CB}-\mathrm{V}$, \\
$\mathrm{N}=15$ \\
FBS $>145.9$
\end{tabular} & $204.3 \pm 16.3$ & $\begin{array}{l}168.2 \pm 19.9 \\
a * *\end{array}$ & $\begin{array}{l}145.7 \pm 35.2 \\
a^{* *} b^{*}\end{array}$ & $\begin{array}{l}148.6 \pm 11.8 \\
a^{* *} b^{*}\end{array}$ & $195.2 \pm 41.2$ & $\begin{array}{l}131.7 \pm 16.6 \\
a^{* *}\end{array}$ & $\begin{array}{l}118.2 \pm 27.1 \\
a^{* *}\end{array}$ & $\begin{array}{l}123.1 \pm 28.9 \\
a * *\end{array}$ & $33.5 \pm 4.1$ & $\begin{array}{l}42.8 \pm 10.3 \\
a^{*}\end{array}$ & $41.9 \pm 19.5$ & $\begin{array}{l}52 \pm 30 \\
a^{*}\end{array}$ \\
\hline
\end{tabular}


Table 4: Effect of Diabegon ${ }^{\circledR}$ on VLDL \& LDL level in MetS with Non-insulin dependent diabetes mellitus subjects of different age groups (N=58)

\begin{tabular}{|c|c|c|c|c|c|c|c|c|}
\hline \multirow[b]{2}{*}{ (Group) } & \multicolumn{4}{|c|}{ VLDL (mg/dl) } & \multicolumn{4}{|l|}{ LDL (mg/dl) } \\
\hline & Initial (a) & 6 month of therapy (b) & $\begin{array}{l}12 \text { month } \\
\text { of therapy(c) }\end{array}$ & $\begin{array}{l}18 \text { month } \\
\text { of therapy (d) }\end{array}$ & Initial (a) & 6 month of therapy (b) & $\begin{array}{l}12 \text { month } \\
\text { of therapy(c) }\end{array}$ & $\begin{array}{l}18 \text { month } \\
\text { of therapy (d) }\end{array}$ \\
\hline \begin{tabular}{|l|} 
Group I \\
$(35-50), \mathrm{N}=10$
\end{tabular} & $56.2 \pm 18.7$ & $38.4 \pm 6.3 \mathrm{a}^{*}$ & $22 \pm 1.76 \mathrm{a}^{* *} \mathrm{~b}^{* *}$ & $25.2 \pm 7.57 \mathrm{a}^{* *} \mathrm{~b}^{* * *}$ & $113.7 \pm 30.2$ & $87 \pm 55.9$ & $71.9 \pm 15 \mathrm{a}^{* *}$ & $51.7 \pm 19 \mathrm{a}^{* *} \mathrm{c}^{*}$ \\
\hline \begin{tabular}{|l} 
Group II \\
$(51-65), N=34$
\end{tabular} & $33.8 \pm 5.8$ & $24 \pm 11.3 \mathrm{a}^{* *}$ & $24.2 \pm 3.2 \mathrm{a}^{* *}$ & $25.1 \pm 9.4 \mathrm{a}^{* * *}$ & $89.5 \pm 23$ & $127.1 \pm 24.9 \mathrm{a}^{* *}$ & $106.2 \pm 42.3 \mathrm{a}^{*} \mathrm{~b} *$ & $64 \pm 41.7 \mathrm{a}^{*} \mathrm{~b}^{* *} \mathrm{c} * *$ \\
\hline Group III $>65, N=14$ & $26.7 \pm 6.6$ & $31.4 \pm 2.8 \mathrm{a}^{*}$ & $27.3 \pm 8.6$ & $20.2 \pm 9.3 \mathrm{a}^{*} \mathrm{~b} * \mathrm{c}^{*}$ & $109.2 \pm 20.1$ & $100.5 \pm 56.8$ & $96 \pm 7.1 \mathrm{a}^{*}$ & $75 \pm 15.4 \mathrm{a}^{* *} \mathrm{c}^{* *}$ \\
\hline $\begin{array}{l}\text { CB-IV, N=43 } \\
\text { FBS }<145.9\end{array}$ & $27 \pm 18.5$ & $26.3 \pm 9.9$ & $22.9 \pm 10.1$ & $21.7 \pm 13 b^{*}$ & $90.3 \pm 22.6$ & $110.9 \pm 41.1 \mathrm{a}^{*}$ & $108.7 \pm 51.3 \mathrm{a}^{*}$ & $70 \pm 40 a^{*} b * c * *$ \\
\hline $\begin{array}{l}\text { CB-V, } N=15 \\
\text { FBS }>145.9\end{array}$ & $39.04 \pm 8.24$ & $26.3 \pm 3.3 \mathrm{a}^{* *}$ & $22 \pm 4.4 a^{* *} b^{*}$ & $28.7 \pm 5.3 \mathrm{a}^{* *} \mathrm{c}^{* *}$ & $131.77 \pm 12.1$ & $198.9 \pm 6.28 \mathrm{a}^{* *}$ & $96.07 \pm 11.2 \mathrm{a}^{* *}$ & $61 \pm 8.2 \mathrm{a}^{* *} \mathrm{~b}^{* *} \mathrm{c}^{* * *}$ \\
\hline
\end{tabular}

FBS, fasting blood sugar; CB, Combination, data were analyzed by ANOVA Bonferroni t-test. Levels of significance were represented in the form of ${ }^{\text {bcdefgh }} \mathrm{P}<0.001(* *)$, bcdefgh $\mathrm{P}<0.05(*)$ when compared with initial (a) group. 
Table 5: Effect of Diabegon ${ }^{\circledR}$ on kidney functions in MetS with Non-insulin dependent diabetes mellitus subjects of different age groups (N=58)

\begin{tabular}{|c|c|c|c|c|c|c|c|c|c|c|c|c|}
\hline \multirow[b]{2}{*}{ (Group) } & \multicolumn{4}{|c|}{ Urea $(\mathrm{mg} / \mathrm{dl})$} & \multicolumn{4}{|c|}{ Uric acid (mg/dl) } & \multicolumn{4}{|c|}{ Creatinine (mg/dl) } \\
\hline & Initial (a) & $\begin{array}{l}6 \text { month of } \\
\text { therapy (b) }\end{array}$ & $\begin{array}{l}12 \text { month } \\
\text { of } \\
\text { therapy(c) }\end{array}$ & $\begin{array}{l}18 \text { month } \\
\text { of therapy(d) }\end{array}$ & $\begin{array}{l}\text { Initial } \\
\text { (a) }\end{array}$ & $\begin{array}{l}6 \text { month of } \\
\text { therapy (b) }\end{array}$ & $\begin{array}{l}12 \text { month } \\
\text { of therapy(c) }\end{array}$ & $\begin{array}{l}18 \text { month } \\
\text { of therapy (d) }\end{array}$ & $\begin{array}{l}\text { Initial } \\
\text { (a) }\end{array}$ & $\begin{array}{l}6 \text { month of } \\
\text { therapy (b) }\end{array}$ & $\begin{array}{l}12 \text { month } \\
\text { of therapy(c) }\end{array}$ & $\begin{array}{l}18 \text { month } \\
\text { of therapy (d) }\end{array}$ \\
\hline \begin{tabular}{|l|} 
Group I \\
$(35-50), \mathrm{N}=10$
\end{tabular} & $20.4 \pm 7.4$ & $23.1 \pm 10.1$ & $25.6 \pm 3.3$ & $23.9 \pm 4.5$ & $5.65 \pm 1.7$ & $4.9 \pm 2.7$ & $4.8 \pm 1.1$ & $5.1 \pm .2$ & $1.6 \pm .59$ & $1.2 \pm .38$ & $\begin{array}{l}0.5 \pm .13 \\
a^{* *} b^{* *}\end{array}$ & $\begin{array}{l}0.74 \pm .12 \\
a * * b * c *\end{array}$ \\
\hline $\begin{array}{l}\text { Group II } \\
(51-65), N=34\end{array}$ & $30 \pm 5.9$ & $17.6 \pm 2.9 \mathrm{a}^{* * *}$ & $\begin{array}{l}25.6 \pm 5.9 \\
a * b * *\end{array}$ & $\begin{array}{l}21.1 \pm 4.3 \\
a^{* *} b^{* *} c^{* *}\end{array}$ & $6.0 \pm 2.1$ & $5.5 \pm 2.7$ & $6.1 \pm 2$ & $\begin{array}{l}7.6 \pm .66 \\
a^{* *} b^{* *} c^{* *}\end{array}$ & $1.0 \pm .106$ & $0.91 \pm .38$ & $\begin{array}{l}0.805 \pm .27 \\
a^{* *}\end{array}$ & $\begin{array}{l}1 \pm .13 \\
c^{* *}\end{array}$ \\
\hline \begin{tabular}{|l} 
Group III $>65$, \\
$N=14$
\end{tabular} & $27.4 \pm 5.8$ & $26.6 \pm .2$ & $\begin{array}{l}21.9 \pm 1.5 \\
a^{*} b^{* *}\end{array}$ & $21.3 \pm 10.5$ & $7.2 \pm .47$ & $\begin{array}{l}6.2 \pm 1.2 \\
a^{*}\end{array}$ & $\begin{array}{l}4.3 \pm 1.5 \\
a^{* *} b^{* *}\end{array}$ & $\begin{array}{l}5.7 \pm 1.9 \\
a * c^{*}\end{array}$ & $1.17 \pm .86$ & $1.1 \pm .52$ & $\begin{array}{l}0.83 \pm .2 \\
b^{*}\end{array}$ & $\begin{array}{l}0.6 \pm .4 \\
a * b *\end{array}$ \\
\hline $\begin{array}{l}\text { CB-IV, N=43 } \\
\text { FBS }<145.9\end{array}$ & $35.4 \pm 11.5$ & $28 \pm 2$ & $\begin{array}{l}24.2 \pm 5.2 \\
a * * b * *\end{array}$ & $25.1 \pm 5.2 \mathrm{a}^{* *} \mathrm{~b}^{* *}$ & $4.9 \pm 1.1$ & $\begin{array}{l}6.9 \pm 1.7 \\
a * *\end{array}$ & $\begin{array}{l}5.5 \pm .90 \\
a * * b * *\end{array}$ & $\begin{array}{l}6.4 \pm 1.8 \\
a * * c^{*}\end{array}$ & $1.4 \pm .67$ & $\begin{array}{l}1.1 \pm .37 \\
a^{*}\end{array}$ & $\begin{array}{l}0.8 \pm .29 \\
a * * b * *\end{array}$ & $\begin{array}{l}0.8 \pm .5 \\
a * * b^{* *}\end{array}$ \\
\hline $\begin{array}{l}\mathrm{CB}-\mathrm{V}, \mathrm{N}=15 \\
\text { FBS }>145.9\end{array}$ & $23.3 \pm 7.3$ & $\begin{array}{l}27.6 \pm 1.2 \\
a^{*}\end{array}$ & $25.4 \pm 4.6$ & $27 \pm 16$ & $8.8 \pm .98$ & $\begin{array}{l}6.7 \pm .77 \\
a * *\end{array}$ & $\begin{array}{l}5.9 \pm .7 \\
a * * b * *\end{array}$ & $\begin{array}{l}5.7 \pm 1.7 \\
\mathrm{a}^{*} \mathrm{~b}^{*}\end{array}$ & $.96 \pm .44$ & $0.84 \pm .32$ & $0.97 \pm .58$ & $0.70 \pm .26$ \\
\hline
\end{tabular}

Table 6: Effect of Diabegon $®$ on liver functions in MetS with Non-insulin dependent diabetes mellitus subjects of different age groups (N=58)

\begin{tabular}{|c|c|c|c|c|c|c|c|c|c|c|c|c|}
\hline \multirow[b]{2}{*}{ (Group) } & \multicolumn{4}{|c|}{ SGOT (IU/L) } & \multicolumn{4}{|c|}{ SGPT (IU/L) } & \multicolumn{4}{|c|}{ Total Bilirubin (mg/dl) } \\
\hline & $\begin{array}{l}\text { Initial } \\
\text { (a) }\end{array}$ & $\begin{array}{l}6 \text { month of } \\
\text { therapy (b) }\end{array}$ & $\begin{array}{l}12 \text { month } \\
\text { of therapy(c) }\end{array}$ & $\begin{array}{l}18 \text { month } \\
\text { of therapy (d) }\end{array}$ & Initial (a) & $\begin{array}{l}6 \text { month of } \\
\text { therapy (b) }\end{array}$ & $\begin{array}{l}12 \text { month } \\
\text { of therapy(c) }\end{array}$ & $\begin{array}{l}18 \text { month } \\
\text { of therapy (d) }\end{array}$ & 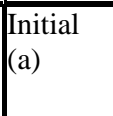 & $\begin{array}{l}6 \text { month of } \\
\text { therapy (b) }\end{array}$ & $\begin{array}{l}12 \text { month } \\
\text { of } \\
\text { therapy(c) }\end{array}$ & $\begin{array}{l}18 \text { month } \\
\text { of therapy (d) }\end{array}$ \\
\hline \begin{tabular}{|l|} 
Group I \\
$(35-50), \mathrm{N}=10$
\end{tabular} & $29.2 \pm 9.8$ & $14.9 \pm 3.2 \mathrm{a}^{* *}$ & $\begin{array}{l}23.9 \pm 2 \\
b^{* *}\end{array}$ & $\begin{array}{l}20.5 \pm 7 \\
a^{*} b^{*}\end{array}$ & $31.5 \pm 12.1$ & $1 \mid \begin{array}{l}19.5 \pm 9.1 \\
a^{*}\end{array}$ & $\begin{array}{l}29.9 \pm 4 \\
b^{*}\end{array}$ & $28.2 \pm 18$ & $0.6 \pm .05$ & $\begin{array}{l}0.7 \pm .10 \\
a^{*}\end{array}$ & $\begin{array}{l}0.76 \pm 0.14 \\
\text { a* }\end{array}$ & $\begin{array}{l}0.65 \pm .05 \\
a * c *\end{array}$ \\
\hline \begin{tabular}{|l} 
Group II \\
$(51-65), N=34$
\end{tabular} & $24.6 \pm 2.3$ & $\begin{array}{l}30 \pm 8.7 \\
a * *\end{array}$ & $\begin{array}{l}32.3 \pm 3.1 \\
a^{* *} b^{*}\end{array}$ & $\begin{array}{l}26.4 \pm 10.2 \\
b^{* *} c^{*}\end{array}$ & $29.4 \pm 1.9$ & $30.1 \pm 7$ & $\begin{array}{l}32.8 \pm 20.8 \\
b^{*}\end{array}$ & $\begin{array}{l}16.3 \pm 3.2 \\
a * * b * * c^{* *}\end{array}$ & $0.81 \pm .34$ & $0.72 \pm .19$ & $\begin{array}{l}0.94 \pm .45 \\
b^{*}\end{array}$ & $\begin{array}{l}0.52 \pm .26 \\
a * * b * * c * *\end{array}$ \\
\hline $\begin{array}{l}\text { Group III }>65, \\
N=14\end{array}$ & $24.9 \pm 3.8$ & $32.2 \pm 5.2 \mathrm{a}^{* *}$ & $\begin{array}{l}30.9 \pm 2 \\
a^{* *}\end{array}$ & $\begin{array}{l}21.7 \pm 3.7 \\
a * b * * c * *\end{array}$ & $29.9 \pm 5.7$ & $32.9 \pm 2.3$ & $30.7 \pm 6.3$ & $\begin{array}{l}20.8 \pm 8.5 \\
a * b * * c^{*}\end{array}$ & $0.74 \pm .06$ & $\begin{array}{l}0.83 \pm .12 \\
\mathrm{a}^{*}\end{array}$ & $\begin{array}{l}0.9 \pm .1 \\
a^{* *}\end{array}$ & $\begin{array}{l}0.66 \pm .15 \\
b * c * *\end{array}$ \\
\hline \begin{tabular}{|l|} 
CB-IV, $N=43$ \\
FBS $<145.9$
\end{tabular} & $29.2 \pm 7.8$ & $\begin{array}{l}18.1 \pm 3 \\
a * *\end{array}$ & $30.2 \pm 3.7$ & $\begin{array}{l}24.5 \pm 6.3 \\
a * b * * c * *\end{array}$ & $33.9 \pm 9.8$ & $\begin{array}{l}16 \pm 4.4 \\
a^{* *}\end{array}$ & $\begin{array}{l}27.3 \pm 3.07 \\
a * b^{* * *}\end{array}$ & $\begin{array}{l}17.6 \pm 3.3 \\
a * b{ }^{*} c * *\end{array}$ & $0.84 \pm .08$ & $\begin{array}{l}0.71 \pm .18 \\
a^{* *}\end{array}$ & $\begin{array}{l}0.91 \pm .42 \\
b^{*}\end{array}$ & $0.67 \pm .22 \mathrm{a}^{* *} \mathrm{c}^{*}$ \\
\hline $\begin{array}{l}\text { CB-V, } N=15 \\
\text { FBS }>145.9\end{array}$ & $22.7 \pm 2.3$ & $\begin{array}{l}30.3 \pm 6 \\
a^{* *}\end{array}$ & $\begin{array}{l}23.8 \pm 6.8 \\
b^{*}\end{array}$ & $\begin{array}{l}15.8 \pm 3.2 \\
\mathrm{a} * * \mathrm{~b} * * \mathrm{c} * *\end{array}$ & $25.5 \pm 5.9$ & $\begin{array}{l}32.8 \pm 4.3 \\
a^{* *}\end{array}$ & $\begin{array}{l}26.8 \pm 1.3 \\
b^{* *}\end{array}$ & $\begin{array}{l}22.1 \pm 16 \\
b^{*}\end{array}$ & $0.83 \pm .03$ & $\begin{array}{l}0.54 \pm .3 \\
a^{*}\end{array}$ & $\begin{array}{l}0.71 \pm .03 \\
a^{* *}\end{array}$ & $\begin{array}{l}0.85 \pm .02 \\
a * b * c * *\end{array}$ \\
\hline
\end{tabular}

FBS, fasting blood sugar; CB, Combination, data were analyzed by ANOVA Bonferroni t-test. Levels of significance were represented in the form of ${ }^{\text {bcdefgh }} \mathrm{P}<0.001(* *),{ }^{\text {bcdefgh }} \mathrm{P}<0.05(*)$ when compared with initial (a) group. 


\section{Effect of diabegon ${ }^{\circledR}$ on blood glucose level in metabolic syndrome with type 2 diabetic subjects $(\mathrm{N}=58)$}

In Group I, when comparison was made between initial and to 18 of therapy, $23.9 \%$ reduction was observed in fasting blood glucose and the reduction was found to be significant (Table 2). When comparisons were made in group II from initial to 6, 9,12,15,18 months of therapies, reduction with $16.6 \%, 19.9 \%, 21.2 \%, 18.2 \%, 26.6 \%$ with significance $(\mathrm{P} \leq .001)$ was seen in fasting blood glucose level. The percentage reduction was found to be $12.3 \%$ after 18 month of therapy in group III. For Group IV, Fasting blood group was significantly reduces when comparison was made between 6 \& 18 months of therapy. $8.1 \%$ of decrease in fasting blood glucose level was seen after 18 month of therapy from its initial level. For Group V, significant $(\mathrm{P} \leq .001)$ reduction was seen after therapy. In P.P blood glucose of group I, significant reduction was observed on $9,12,15$, and 18 month of therapy at a level $19 \%, 32.4 \%, 27.2 \%$, and $22.3 \%$ respectively. For group II, the comparisons made between initial to 6,12 , and 18 month of therapy showed significance $(\mathrm{P} \leq .05)$ with $11.2 \%, 19.3 \%$ and $17.7 \%$ decrease respectively. The therapy showed a significant value in group III $(\mathrm{P}<0.05)$ while comparing initial with 12,18 of therapy with a reduction of $21.7 \%, 26 \%$ respectively. For Group IV, significant reduction was observed in different time interval. Group $\mathrm{V}$, When comparison was made between initial and 12 , and 18 month of therapy significance $(\mathrm{P}<0.05)$ was found with $15.4 \%, 21.4 \%$ reduction in level respectively.

\section{Effect of diabegon $®$ on lipid profile (Total cholesterol, triglyceride, HDL-C, VLDL, LDL) level in metabolic syndrome with non-insulin dependent diabetes mellitus subjects of different age groups $(\mathrm{N}=58)$}

Table 3 represents effect of diabegon therapy on lipid profile. A significant reduction in cholesterol after 6 to 18 month of therapy was seen. When comparison was made between initial to $12 \& 18$ month of therapy for group II, a reduction with $8.3 \%$ and $11 \%$ were observed. Cholesterol level was reduced up to 8.9-14.5\% after 18 month of therapy in group III\&IV. For group V, Comparison between initial to 6, 12 and 18 month of therapy revealed reduction with $17.6 \%, 28.6 \%$ and $27.2 \%$. Triglyceride level in group I showed a significant reduction of 50-60\%. For group II, when comparisons were made between initial to $6,12,18$ month of therapy reduction of $28.9 \%, 28.5 \%$ and $34 \%$ were observed. The triglyceride level in group III was within the normal range during the study. A reduction in triglyceride observed with $27.4 \%$ and $27.8 \%$ in group IV after the therapy. A remarkable reduction was observed in group V. HDL-C level in group I showed 95\% increase after treatment for 18 month. In group II, when initial value was compared with 6 and 18 month of therapy, a slight increase of 17.9-26.8\% were seen. When initial value of group III and IV in HDL-C level was compared, elevation of $6-25.7 \%$ had been observed in different intervals of therapy. An increase in group V for HDL-C was observed with $27.7 \%$ and $55.2 \%$ when compared with initial to 6 and 18 month of therap. For the VLDL in group I showed a reduction of $31.6 \%$ to $60.8 \%$ in different point of therapy. Reduction of $28.9 \%, 28.4 \%$ and $25.7 \%$ in VLDL after 6, 12 and 18 month of therapy in group II. After 6, 12 and 18 month of diabegon therapy yielded significance $(\mathrm{P}<0.001)$ with $32.5 \%, 43.5 \%$ and $26.4 \%$ reduction in group V. Subject in group I compared between initial to $12 \& 18$ month of therapy, $36.7 \%$ and $54.5 \%$ reduction were observed in LDL level. For group III, when initial value was compared with 12 and 18 month of therapy, a reduction was seen with $12 \%$ and $31.3 \%$. The baseline value in Group II and IV was found to be normal and it maintained during the therapy. For group V, when initial value was compared with 6, 12 and 18 month of therapy, a reduction $24.9 \%, 27 \%$ and $53.6 \%$ observed.

\section{Effect of diabegon ${ }^{\circledR}$ on kidney function (urea, uric acid, creatinine) level in metabolic syndrome with non-insulin dependent diabetes mellitus subjects of different age groups $(\mathrm{N}=58)$}

Diabegon therapy on urea, uric acid, creatinine level in T2 diabetes with metabolic syndrome is shown in Table 5. The urea level of the study subjects were found to be normal at base line which was maintain during the therapy in all groups of the subjects. Uric acid level was found to be normal at initial therapy which was seems to be constant in group I, II and IV. For group III, when initial values were compared with 6 and 18 month of therapy, reduction of $13.8 \%$ and $20.8 \%$ was observed. Significant was also observed after 12 month of therapy. In group V, when subjects were compared between initial to 6,12 , and 18 month of therapy reduction of $23.4 \%, 32.9 \%$ and $35.2 \%$ were seen.

Creatinine in the studied subject was within the normal range except group I. For group I, when initial value was compared with 12 and 18 month of therapy, $68.7 \%$ and $53.7 \%$ reduction was observed which was found to be significant. Significant reduction was seen between the different duration of therapy.

\section{Effect of diabegon ${ }^{\circledR}$ on liver function (SGOT, SGPT, Bilirubin) level in metabolic syndrome with non-insulin dependent diabetes mellitus subjects of different age groups $(\mathrm{N}=58)$}

Table 6. Showed the diabegon therapy on liver enzyme in human T2 diabetic subject. After observing the result in the table, we could find that the three enzymes that associated with diabetic complication of liver were at normal range that maintained during the all study follow-up. There was slight elevation and depression in the value but it was within the range of normal value.

\section{Discussion}

Combination of metabolic syndrome variables with type 2 diabetes leads to more complex in term of the risk factor for mortality. Our study recruited these subjects with essentially required a multifactorial intervention including treatment of arterial hypertension and dyslipidaemia to prevent cardiovascular complications. In recent years the popularity of complementary medicine has increased. Much interest has been focused on exploring herbal preparations. Our study showed an Overall reduction in the individual parameter in metabolic syndrome subject and highly significant in the $35-50$ years age group (Table 2). The reduction in fasting blood, triglyceride in the age group 35-50 years $21 \%$, 49.1\%. The HDL-cholesterol level was increased by $56 \%$. The overall reduction in fasting blood, triglyceride in the age group 51-65 years $21.3 \%, 30.4 \%$. The HDL-cholesterol level was increased by $17 \%$. In the $>65$ years age group the reduction was $10 \%, 20 \%$. The elevation in HDL-C levels was $12 \%$. Generally, the reduction in fasting blood glucose range from $12.3 \%(\mathrm{P}<0.05)$ to $42 \%(\mathrm{P}<0.001)$ after 18 month of therapy while in post prandial blood glucose, the decrease ranged from $28 \%(\mathrm{P}<0.05)$ to $32 \%(\mathrm{P}<0.05)$ after 18 month of therapy (Table 2). Earlier study showed that the Oral administration of diabegon delayed the induction of glucose intolerance and euglycemia in high fructose diet fed rats. Diabegon treatment inhibits the disturbance in glucose metabolism in the liver by reducing the glycogen accumulation in liver, which might be due to induced glycogenolysis and/ or inhibited gluconeogenesis (Hariom et al., 2007).The Momordica charantia (bittermelon) fruits, seeds and seedlings have various compounds with antidiabetic properties (Basch et al., 2003). Administration of $230 \mathrm{~g} /$ day of momordica for 8-11 weeks to a group of nine diabetic patients, significantly improved 
the results of oral glucose tolerance (Leatherdale et al., 1981). New studies suggest bitter gourd has the ability to regenerate dormant pancreatic beta cells that stimulate insulin in the body (Saxena et al., 2004). Gymnema sylvestrae have a series of gemnemic acids that have antidiabetic properties.

The aqueous extract of Gymnema sylvestrae improved glucose control and insulin requirement in IDDM and NIDDM subjects (Shanmugasundaram et al., 1990). Trigonella foenum graecum (also known as fenugreek) has an alkaloid trigonelline which posses hypoglycemic properties (Grover et al., 2002). Fenugreek significantly lowered the fasting blood glucose and glucose intolerance (Sharma et al., 1990). The green and crude leaf extract of Aegle marmelos exhibited hypoglycemic potential in alloxan induced diabetes (Ponnachan et al., 1993). The ethanolic extract of Eugenia jambolana seed on hyperglycemia has been reported. Curcuma longa and Emblica officinalis contain various nutraceuticals such as terpenoids, curcuminoids and polyphenols/flavonoids respectively, they are all endowed with biological effects such as antioxidant, antidiabetic, immunomodulatory and hypolipidemic properties. The shilajit is reported to possess antihyperglycaemic and antilipidemic potentials when checked in alloxan induced diabetic rats (Trivedi et al., 2004). Liver and kidney functions were within the normal range at baseline in most groups. In the present investigation diabetic subjects with metabolic syndrome when administered with diabegon exhibited a significant decrease in lipid profile. In our study cholesterol level decreased from $11 \%$ to $27.2 \%(\mathrm{P}<0.001)$ after 18 months of therapy. The percentage decrease in triglyceride level ranged from $24 \%$ to $55 \%$. The HDL-C level increased in all groups of subjects (Table 3). The VLDL and LDL level reduced up to $60 \%$ \& $54 \%$ respectively (Table 4 ). The level of HDL-cholesterol increased after diabegon therapy. This indicates that diabegon may help to increase transport of peripheral tissue cholesterol to liver and thereby decrease blood cholesterol level. The liver and kidney function tests were also significantly improved after the therapy. The urea, uric acid, creatinine decreases up to $30-60 \%$ in higher baseline values in groups and constantly maintained during the therapy (Table 5). The SGOT, SGPT \& bilirubin levels showed 20 to $40 \%$ of reduction in group II and group IV. The liver functions of the recruited subject were normal at the baseline.

On the basis of results, it could be concluded that oral administration of diabegon, a polyherbal formulation has antihyperglycemic and antilipidemic potential for long term treatment with great promises for metabolic syndrome. The study also revealed that therapy does not show any toxic effect on liver and kidney. The herbal formulation considered as safe therapy for a long term and effective management of type II diabetes as well as metabolic syndrome.

\section{Acknowledgements}

The study was supported in part by research grants from university grants commission, New Delhi and Biotechnology Council of Madhya Pradesh, Bhopal. Thanks are also due to Deendayal Research Foundation for providing medicine for the study.

\section{References}

1. Babu, P.S., and Stanely, P.P. (2004). Antihyperglycaemic and antioxidant effect of hyponidd, an ayurvedic herbomineral formulation in streptozotocin-induced diabetic rats. J. Pharm Pharmacol. 56: 1435-1442.

2. Balkau, B., Charles, M.A., Drivsholm, T., Borch-Johnsen, K., Wareham, N., and Yudkin, J.S. (2002). The European Group for the Study of Insulin Resistance (EGIR) Frequency of WHO-defined metabolic syndrome in European cohort and an alternative definition of an insulin resistance syndrome. Diabetes Metab. 28: 364-376.

3. Basch, E., Gabardi, S., and Ulbricht, C. (2003). Bitter melon (Momordica Charentia): A review of efficacy and safety. Am J Health Syst Pharm. 60: 356-359

4. Bhandari, U., Kanojia, R., and Pillai, K.K. (2005). Effect of ethanolic extract of Zingiber officinale on dyslipidaemia in diabetic rats. J. Ethnopharmacol. 97: 227-230.

5. Bowers, L.D. (1980). Kinetic serum creatinine assays I. The role of various factors in determining specificity. Clin. Chem. $26: 551-554$.

6. Burtis, C.A., and Ashwood, E.R. (1999). Tietz textbook of clinical chemistry. $3^{\text {rd }}$ ed. Philadelphia: B Saunders Company pp. 617-721.

7. Chen, J., Muntner, P., Hamm, L.L., Fonseca, V., Batuman, V., Whelton, P.K., and He, J. (2003). Insulin resistance and risk of CKD in nondiabetic US adults. J Am Soc Nephrol. 14: 469-477.

8. Eckel, R.H., Grundy, S.M., and Zimmet, P.Z. (2005). The metabolic syndrome. Lancet 365: 1415-1428.

9. Executive Summary of the Third Report of National Cholesterol Education Program (NCEP 2001) Expert Panel on Detection, Evaluation, and Treatment of High Blood Cholesterol in Adults (Adult Treatment Panel III). JAMA 285: 2486-2497.

10. Fawcett, J.K., and Scott, J.E. (1960). Determination of urea in serum by GLDH method. J.Chim. Pathol. 13:156.

11. Ford, E.S., Giles, W.H., and Dietz, W.H. (2002). Prevalence of the metabolic syndrome among US adults: Findings from the 3rd national health and nutrition examination survey. J Am Med Assoc. 287: 356-359.

12. Fossati, P., and Prencipe, L. (1982). Serum triglycerides determined colorimetrically with an enzyme that produces hydrogen peroxide. Clin. Chem. 28: 2077-2080.

13. Fossati, P., Prencipe, L., and Berti, G. (1980). Use of 3,5-dichloro-2- hydroxybenzenesulfonic acid/4-aminophenazone chromogenic system in direct enzymic assay of uric acid in serum and urine. Clin Chem. 26: 227-231.

14. Grover, J.K., Yadav, S., and Vats, V. (2002). Medicinal plants of India with antidiabetic potential. J Ethnopharmacol. 81: 81-100.

15. Grundy, S.M. (2006). Does the Metabolic Syndrome have Value in Clinical Practice? Am J Clin Nutr. 83: 1248-1251.

16. Halim, E.M., and Hussain, A. (2002). Hypoglycemic, hypolipidemic and antioxidant properties of combination of curcumin from curcuma longa, linn, and partially purified product from abroma augusta, linn. In streptozotocin induced diabetes. Indian J Clin Biochem. 17: 33-43.

17. Jendrassik, L., and Grof, P. (1938). Colorimetric method for estimation of serum bilirubin. Biochemische Zeitschrift 2: 81.

18. Leatherdale, B.A., Panesar, R.K., and Singh, G. (1981). Improvement in glucose tolerance due to Momordica charantia (karela). Br Med J. 282: 1823-1824.

19. Lopes-Virella, M.F., Stone, P., Ellis, S., and Colwell, J.A. (1977). Cholesterol determination in high-density lipoproteins separated by three different methods. Chem Communications 23: $882-883$.

20. Novakovic, B., and Popovic, M. (2001). Occurrence of the metabolic syndrome in the population of the town of Novi Sad. Med Pregl. 54: 17-20.

21. Ponnachan, P.T., Paulose, C.S., and Panikkar, K.R. (1993). Effect of leaf extract of Aegle marmelose in diabetic rats. Indian J Exp Biol. 31: 345347.

22. Reaven, G.M. (1988). Banting Lecture. Role of insulin resistance in human disease. Diabetes 37: 1595-1607. 
23. Saxena, A., and Vikram, N.K. (2004). Role of selected Indian plants in management of type 2 diabetes: a review. J Altern Complement Med. 10: 369-378.

24. Seshiah (2004). Drug Management of Type 2 Diabetes Mellitus - Clinical Experience at Diabetes Center in South India Year 24: $40-46$.

25. Shanmugasundaram, E.R., Gopinath, K.L., Radha Shanmugasundaram, K., and Rajendran, V.M. (1990). Possible regeneration of the islets of Langerhans in streptozotocin-diabetic rats given Gymnema sylvestre leaf extracts. J. Ethnopharmacol. 30: 265-279.

26. Sharma, R.D., Raghuram, T.C., and Rao, N.S. (1990). Effect of fenugreek seeds on blood glucose and serum lipids in type I diabetes. Eur J Clin Nutr. 44: 301-306.

27. Sone, H., Tanaka, S., Iimuro, S., Oida, K., Yamasaki, Y., Oikawa, S., Ishibashi, S., Katayama, S., Ito, H., Ohashi, Y., Akanuma, Y., and Yamada, N. (2009). Components of metabolic syndrome and their combinations as predictors of cardiovascular disease in Japanese patients with type 2 diabetes. Implications for improved definition. Analysis from Japan Diabetes Complications Study (JDCS). J Atheroscler Thromb. 16: 380-387.

28. Stockbridge, H., Hardy, R.I., and Glueck, C.J. (1989). Public cholesterol screening: motivation for participation, follow-up outcome, selfknowledge, and coronary heart disease risk factor intervention. J Lab Clin. Med. 114: 142-151.

29. Tongia, A., Tongia, S.K., and Dave, M. (2004). Phytochemical determination and extraction of Momordica charantia fruit and its hypoglycemic potentiation of oral hypoglycemic drugs in diabetes mellitus (NIDDM). Indian J Physiol Pharmacol. 48: 241-244.

30. Trinder, P. (1969). Determination of glucose in blood using glucose oxidase with an alternative oxygen acceptor. Analy Clin Biochem. 6: $24-27$.

31. Trivedi, N.A., Mazumdar, B., Bhatt, J.D., and Hemavathi, K.G. (2004). Effect of shilajit on blood glucose and lipid profile in alloxan- induced diabetic rats. Indian J Physiol Pharmacol. 36: 373-376.

32. WHO Technical Report Series No.844 World Health Organization. Geneva 1994.

33. Wilson, P.W., D'Agostino, R.B., Parise, H., Sullivan, L., and Meigs, J.B. (2005). Metabolic syndrome as a precursor of cardiovascular disease and type 2 diabetes mellitus. Circulatn 112: 3066-3072.

34. Yadav, H., Jain, S., Prasad, G.B.K.S., and Yadav, M. (2007). Preventive Effect of Diabegon, a Polyherbal Preparation, During Progression of Diabetes Induced by High-Fructose Feeding in Rats. J Pharmacol Sci. 105: 12-21. 